\title{
Ginsenoside-Rb1 Protects Hypoxic- and Ischemic-Damaged Cardiomyocytes by Regulating Expression of miRNAs
}

\author{
Xu Yan, ${ }^{1,2,3}$ Jinrong Xue, ${ }^{4}$ Hongjin Wu, ${ }^{1}$ Shengqi Wang, ${ }^{5}$ Yuna Liu, ${ }^{3}$ \\ Sidao Zheng, ${ }^{3}$ Chengying Zhang, ${ }^{3}$ and Cui Yang ${ }^{3}$ \\ ${ }^{1}$ Beijing Haidian Hospital, Haidian Section of Peking University Third Hospital, 29 Zhongguancun Dajie, Haidian District, \\ Beijing 100080, China \\ ${ }^{2}$ Postdoctoral Workstation of the Zhongguancun Haidian Science Park, No. 6 Sijiqing Road, Haidian District, Beijing 100195, China \\ ${ }^{3}$ Beijing Hospital of Integrated Traditional Chinese and Western Medicine, Dongjie 3, Yongding Road, Haidian District, \\ Beijing 100039, China \\ ${ }^{4}$ Department of Cardiovascular Surgery, Beijing Aortic Disease Center, Beijing Anzhen Hospital, Capital Medical University, \\ No. 2 Anzhen Road, Chaoyang District, Beijing 100029, China \\ ${ }^{5}$ Beijing Institute of Radiation Medicine, Beijing 100850, China
}

Correspondence should be addressed to Hongjin Wu; whjyuanzhang@aliyun.com

Received 9 July 2014; Revised 25 September 2014; Accepted 2 October 2014

Academic Editor: Hao Xu

Copyright (C) $2015 \mathrm{Xu}$ Yan et al. This is an open access article distributed under the Creative Commons Attribution License, which permits unrestricted use, distribution, and reproduction in any medium, provided the original work is properly cited.

\begin{abstract}
Ginsenoside (GS-Rb1) is one of the most important active compounds of ginseng, with extensive evidence of its cardioprotective properties. However, the miRNA mediated mechanism of GS-Rb1 on cardiomyocytes remains unclear. Here, the roles of miRNAs in cardioprotective activity of GS-Rb1 were investigated in hypoxic- and ischemic-damaged cardiomyocytes. Neonatal rat cardiomyocytes (NRCMs) were first isolated, cultured, and then incubated with or without GS-Rb1 (2.5-40 $\mu \mathrm{M})$ in vitro under conditions of hypoxia and ischemia. Cell growth, proliferation, and apoptosis were detected by MTT and flow cytometry. Expressions of various microRNAs were analyzed by real-time PCR. Compared with that of the control group, GS-Rb1 significantly decreased cell death in a dose-dependent manner and expressions of mir-1, mir-29a, and mir-208 obviously increased in the experimental model groups. In contrast, expressions of mir-21 and mir-320 were significantly downregulated and GS-Rb1 could reverse the differences in a certain extent. The miRNAs might be involved in the protective effect of GS-Rbl on the hypoxia/ischemia injuries in cardiomyocytes. The effect might be based on the upregulation of mir-1, mir-29a, and mir-208 and downregulation of mir-21 and mir-320. This might provide us a new target to explore the novel strategy for ischemic cardioprotection.
\end{abstract}

\section{Introduction}

MicroRNAs (miRNAs) are a kind of conservative single stranded noncoding RNA molecules found in animals, plants, virus, and other organisms and regulate proliferation, differentiation, apoptosis and metabolism, and other cellular processes $[1,2]$. In recent years, investigations found that miRNAs play an important role in the development of cardiovascular diseases and may become a possible new target in the treatment of cardiovascular diseases [3].

Myocardial ischemic injury resulting from severe impairment of the coronary blood supply is a severe stress that leads to the loss of cardiomyocytes by apoptosis and necrosis.
MicroRNAs have been proved to be potential biomarkers for ischemic heart disease, such as mir-1, mir-133, mir-208, and mir-499 [4-6]. Several studies also demonstrated that miRNAs dysregulation has a key role in the ischemic heart disease process. Various miRNAs can regulate gene expression at the posttranscriptional level by either translational repression of a target mRNA or degradation of myocardial death induced mir expression in the genome of most eukaryotes. Many studies have revealed that microRNAs could be the therapeutic targets for common used drugs or new drug design, which could be potentially used as complementary and alternative interventions for the treatment of ischemic heart disease. 
Ginseng, the root of Panax ginseng C.A. Meyer, has been widely used in traditional Chinese medicine for several thousand years [7]. Ginseng plays critical roles in the endocrine, immune, central nervous, and especially the cardiovascular systems [8]. Ginsenoside-Rb1 (GS-Rb1), a major pharmacological extract, is one of the most important active compounds of ginseng, with extensive evidence of its cardioprotective properties. Numerous studies have indicated that the cardioprotective effect of GS-Rbl was affected by multiple pathways [9]. However, regulatory roles of GSRb1 in miRNA during cardiomyocytes apoptosis are rarely studied. In the present investigation, rat myocardial cells were first isolated, cultured, and incubated with GS-Rb1 in vitro under conditions of hypoxia and ischemia. The protective roles of GS-Rb1 were explored, and five circulation-related microRNAs' expression change in each group was then analyzed by poly(A) tailing SYBR Green real-time PCR.

\section{Materials and Methods}

2.1. Materials. GS-Rb1 (catalog number 110704) purchased from National Institutes for Food and Drug Control was dissolved in phosphate-buffered saline (PBS) to create a stock solution for subsequent dilution. miRNA extracting kit (CW0627), reverse transcription kit (CW2141), and fluorescent quantitation PCR (CW2142) kit were all purchased from BeiJing Cowin Biotech Co., Ltd. Annexin V-FITC/PI kit was purchased from Kaiji Biological Engineering Institute.

2.2. Isolation and Culture of Neonatal Rat Cardiomyocytes. All experiments were approved by the Beijing Ethics Committee for the Use of Experimental Animals. Primary cultures of NRCMs from 12-24-hour-old Sprague Dawley rats (Vital River Laboratories, Beijing, China) were prepared by means of gentle serial trypsinization as described before with slight modification [10]. Briefly, the ventricular myocardium was removed and cut into small pieces $\left(1-2 \mathrm{~mm}^{3}\right)$. The ventricles obtained were washed three times in cold PBS and digested 5 times for $5 \mathrm{~min}$ each at $37^{\circ} \mathrm{C}$ with $0.18 \%(\mathrm{w} / \mathrm{v})$ trypsin and $0.01 \%$ EDTA. Addition of an equal volume of cold Dulbecco's modified Eagle's medium (DMEM) containing $10 \%(\mathrm{v} / \mathrm{v})$ newborn calf serum was used to terminate the digestion. Then, cells were collected by centrifugation for $10 \mathrm{~min}$ at $1000 \mathrm{~g} / \mathrm{min}$ at room temperature. Cells were then resuspended in DMEM with 20\% (v/v) FCS for $60 \mathrm{~min}$ to facilitate separation of ventricular myocytes from the fasterattaching nonmyocytes. The NRCMs were then collected and plated in collagen-coated 96- or 6-well plates and maintained at $37^{\circ} \mathrm{C}$ in a $5 \% \mathrm{CO}_{2} / 95 \%$ air humidified incubator in DMEM containing $10 \%(\mathrm{v} / \mathrm{v})$ fetal bovine serum, $100 \mathrm{U} / \mathrm{mL}$ penicillin, and $100 \mathrm{mg} / \mathrm{mL}$ streptomycin. The following experiments used spontaneously beating cardiomyocytes $48-72 \mathrm{~h}$ after plating.

2.3. Hypoxia/Ischemia Treatment. To generate hypoxic/ ischemic conditions, culture medium was replaced with DMEM (no glucose) (Gibco, Grand Island, USA) with serum free. NRCMs with or without GS-Rb1 were then placed in a W-Zip package (an0010, Oxide Anaerobe Pouch System), which was capable of depleting the concentration of $\mathrm{O}_{2}$ down to $10 \%$ in $2 \mathrm{~h}$. The sealed package was subsequently placed into a $37^{\circ} \mathrm{C}$ incubator for $12 \mathrm{~h}$ after pretreatment with GS-Rb1 for $6 \mathrm{~h}$. The control plates were kept in normoxic conditions for the corresponding times.

2.4. MTT Assay. NRCMs viability was determined using the MTT assay. Cardiomyocytes were plated on 96-well dishes at $2 \times 104$ cells/well. MTT at $5 \mathrm{mg} / \mathrm{mL}$ was added to each well immediately after $12 \mathrm{~h}$ of hypoxia/ischemia. Plates were incubated for $4 \mathrm{~h}$ at $37^{\circ} \mathrm{C}$. The medium was aspirated from each well and $100 \mu \mathrm{L}$ of DMSO was added to dissolve the formazan crystals. The optical density of each well was read at 492 nm using a Microplate Reader (Bio-Rad, Hercules, CA). Results are given as percentages of the control group taken as $100 \%$.

2.5. Apoptosis Assay by Annexin V/PI Staining. NRCMs with different concentration of GS-Rb1 exposed to hypoxia/ischemia conditions were harvested and washed with PBS. The percentage of normal nonapoptotic cells was measured by double supravital staining with Annexin V and PI, using an Annexin V Apoptosis Detection kit (KeyGen, Nanjing, China). Flow cytometric analysis used a Cytomics FC500 flow cytometer with CXP software (Beckman Coulter, Fullerton, USA), the operator being blind to the group assignment.

2.6. Detection of miRNA Expression Using Poly(A) Tailing SYBR Green Real-Time PCR. Total RNA $(5 \mu \mathrm{g})$ was treated with DNase I for 15 minutes at $22^{\circ} \mathrm{C}$ (Invitrogen) and then poly(A)-tailed using poly(A) polymerase $(\mathrm{NEB})$ at $37^{\circ} \mathrm{C}$ for 1 hour. The final reaction mixture was extracted with phenol/chloroform, precipitated with isopropanol, and redissolved in $25 \mu \mathrm{L}$ of diethylpyrocarbonate-treated water. Poly(A)-tailed RNA $(6 \mu \mathrm{L})$ was reverse-transcribed into firststrand cDNA using a miRNA cDNA kit (cw2141, BeiJing Cowin Biotech Co., Ltd.). For PCR, 30 ng of cDNA was used as a template in each reaction using miRNA Real-Time PCR Assay Kit (cw2142, BeiJing Cowin Biotech Co., Ltd.). The forward primer for each miRNA was

mir-1: $5^{\prime}$-GCGTGGAATGTAAAGAAGTGTGTAT AAA- $3^{\prime}$;

mir-29a: $5^{\prime}$-TAGCACCATCTGAAATCGGTT AAAA- $3^{\prime}$;

mir-208: 5' -ATAAGACGAGCAAAAAGCAAAAA AAA- $3^{\prime}$;

mir-21: $5^{\prime}$-GCTAGCTTATCAGACTGATGTTG AAAA- $3^{\prime}$;

mir-320: 5' -AAGCTGGGTTGAGAGGGCGA-3' ; U6 small noncoding RNA sequence was amplified as an internal control using the primers $5^{\prime}$ CTCGCTTCGGCAGCACA-3' (forward) and $5^{\prime}$-AACGCTTCACGAATTTGCGT-3' (reverse). 


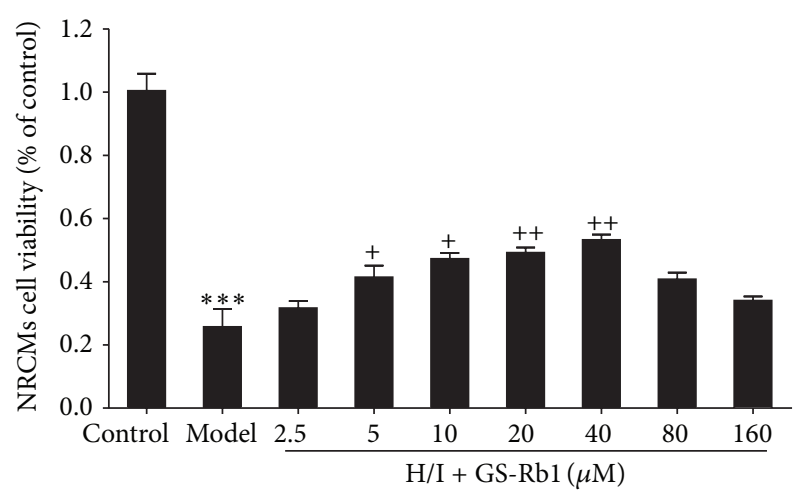

FIGURE 1: Protective effect of GS-Rb1 on H/I-induced NRCMs death. NRCMs were copretreated with or without GS-Rb1 during H/I condition for $12 \mathrm{~h}$. Cell viability was determined by MTT assay. Error bars represent mean \pm SD. Error bars represent mean \pm SD. ${ }^{* * *} P<0.001$ versus control, ${ }^{+} P<0.05$, and ${ }^{++} P<0.01$ versus $\mathrm{H} / \mathrm{I}$ group $(n=3)$.

The anneal temperature was $63^{\circ} \mathrm{C}$. The SYBR Green-based real-time PCR was performed using ABI 7500 Real-Time PCR System (Applied Biosystems). The relative expression of miRNA was calculated based on the formula: $2^{-(\Delta \mathrm{CtmiR}-\Delta \mathrm{CtU} 6)}$.

2.7. Statistical Analysis. The data obtained were presented as the mean \pm SEM of 3 independent experiments. The relationship between two factors was analyzed by Pearson correlation analysis. Bootstrap was used in paired samples tests. Group results were analysed for variance using ANOVA. Two groups were compared by Student's $t$-test. All analyses used GraphPad Prism 5.0 software. A $P<0.05$ indicated that the difference was statistically significant. A $P<0.01$ indicated that the difference was extremely and statistically significant.

\section{Results}

3.1. Effects of GS-Rb1 on Hypoxic- and Ischemic-Induced Damaged Cardiomyocytes. Ginsenoside plays important roles in physiological and pathological conditions of various cells. In this research, neonatal rat cardiomyocytes were treated under hypoxia and ischemic conditions in the presence or absence of GS-Rb1. As shown in Figure 1, addition of GS-Rb1 significantly decreased cell death in a dependent manner at an optimal concentration of $40 \mu \mathrm{M}$, indicating that GS-Rb1 has a protective role in hypoxic- and ischemic-induced damaged myocardial cells.

3.2. Effects of GS-Rb1 on Cell Apoptosis of Cardiomyocytes. To examine the effect of GS-Rbl on the cell death induced by $\mathrm{H} / \mathrm{I}$, Annexin V-FITC /propidium iodide (PI) double-staining assay of cells was analyzed by flow cytometry (Figure 2). The percentage of apoptotic cells (including early and late apoptotic cells) markedly increased in H/I group compared to the control group. With GS-Rbl, apoptosis accounted for $31.0 \pm 1.8 \%$ at $40 \mu \mathrm{M}$ GS-Rb1, with the surviving cells increasing $52.8 \pm 3.1 \%$. These data indicate that GS-Rb1 can inhibit hypoxic- and ischemic-induced cell apoptosis of neonatal rat cardiomyocytes.

3.3. Expression of MicroRNAs in Hypoxic- and IschemicTreated Cardiomyocytes. The level of microRNAs was detected by real-time RT-PCR assay. The expression level of mir-1, mir-29a, and mir-208 was increased in the H/I group (5.9-, 3.4-, and 9.3-fold versus control, relatively), while that of mir-21 and mir-320 was significantly decreased (0.35and 0.41 -fold versus control, relatively). With the treatment of GS-Rb1, the expression change of miRNAs in H/I group could be reversed in a certain extent (Figure 3 ).

\section{Discussion}

GS-Rb1 is one of the most important active compounds of ginseng, and it has multiple pharmacological actions [1114] and the protective effects of different organs [15-20]. Furthermore, GS-Rb1 has been proved to have cardiovascular protective effect by considerable research.

MicroRNAs (miRNAs) are a kind of conservative single stranded noncoding RNA molecules found in animals, plants, virus, and other organisms and are important regulators of gene expression and fundamentally impact on cardiovascular functions. In recent years, investigations found that miRNAs play an important role in the development of cardiovascular diseases and may become a possible new target in the treatment of cardiovascular diseases. Some researchers have revealed that the miRNAs could be the targets of traditional Chinese medicine to protect cardiovascular system. Tanshinone IIA, a lipid-soluble pharmacologically active compound extracted from the rhizome of traditional Chinese herb Salvia miltiorrhiza, has been reported to improve hypoxic cardiac myocytes and postinfarction rat cardiomyocytes by regulating mir-133 and mir-1 and MAPK pathways [21, 22]. In the present research, we have found that GS-Rb1 could protect primary cardiomyocytes from hypoxia and ischemia injuries by reducing cell apoptosis and modulating circulation of miRNAs. Compared with that of the control group, expressions of mir-1, mir-29a, and mir208 obviously increased in the experimental model groups. In contrast, expressions of mir-21 and mir-320 were significantly downregulated and GS-Rbl incubation in model group could reverse the differences in a certain extent.

Specifically, mir-1 is skeletal and cardiac muscle specific microRNA necessary for postmitotic muscle proliferation and differentiation [23]. Many researchers have proved that mir-1 plays an important part during cardiac apoptosis [24, 25]. Overexpression of mir-1 could exacerbate cardiac injury; on the contrary, knockdown of mir-1 significantly attenuated cardiac ischemia/reperfusion injury [26]. Mir-29 has shown relationship with cancer cell apoptosis by activating p53 [27]. Downregulation of mir-29 (mir-29a and mir-29c) by antisense inhibitor also protected $\mathrm{H} 9 \mathrm{c} 2$ cardiomyocytes from simulated IR injury. Antagomirs against mir-29a or mir-29c significantly reduced myocardial infarct size and apoptosis in 

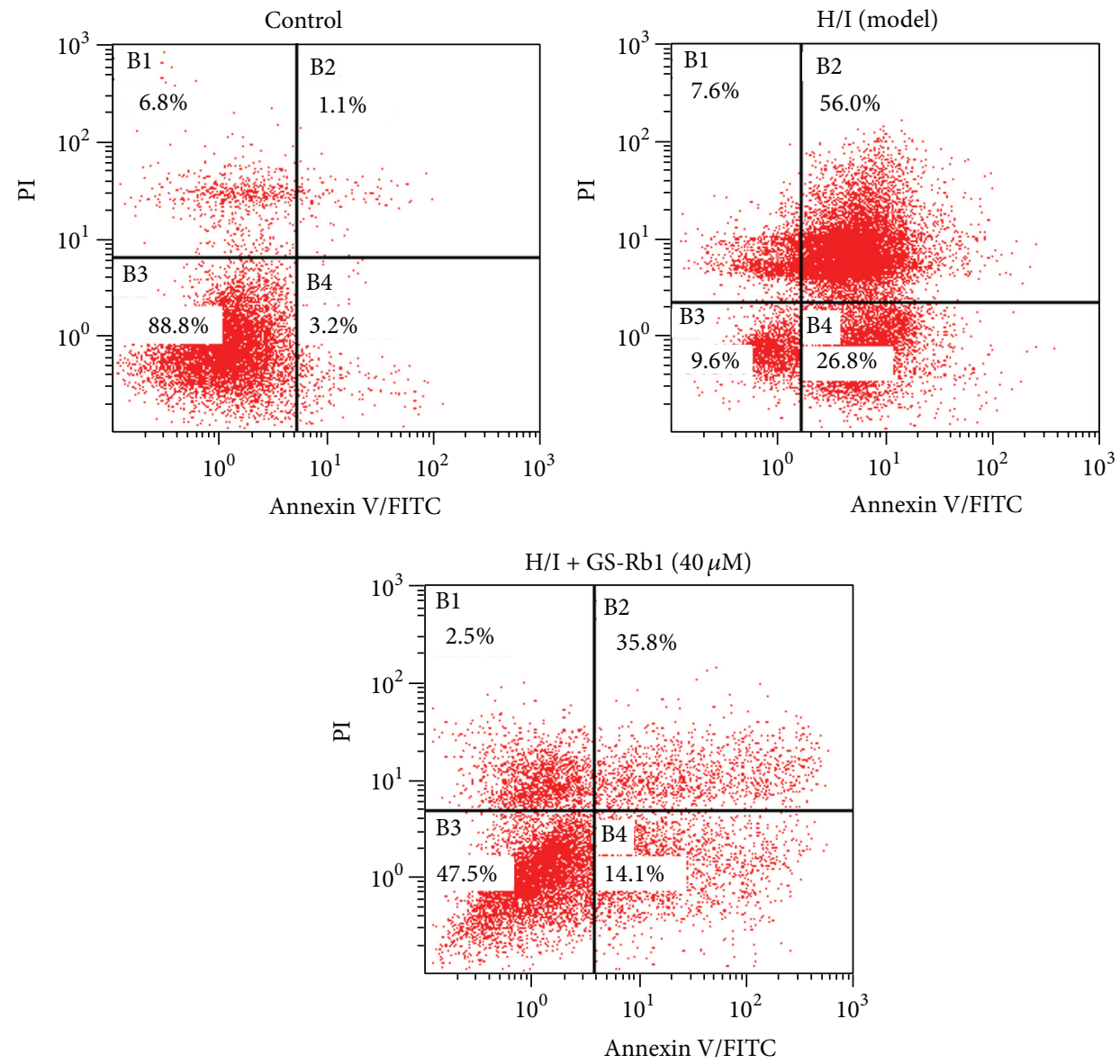

(a)

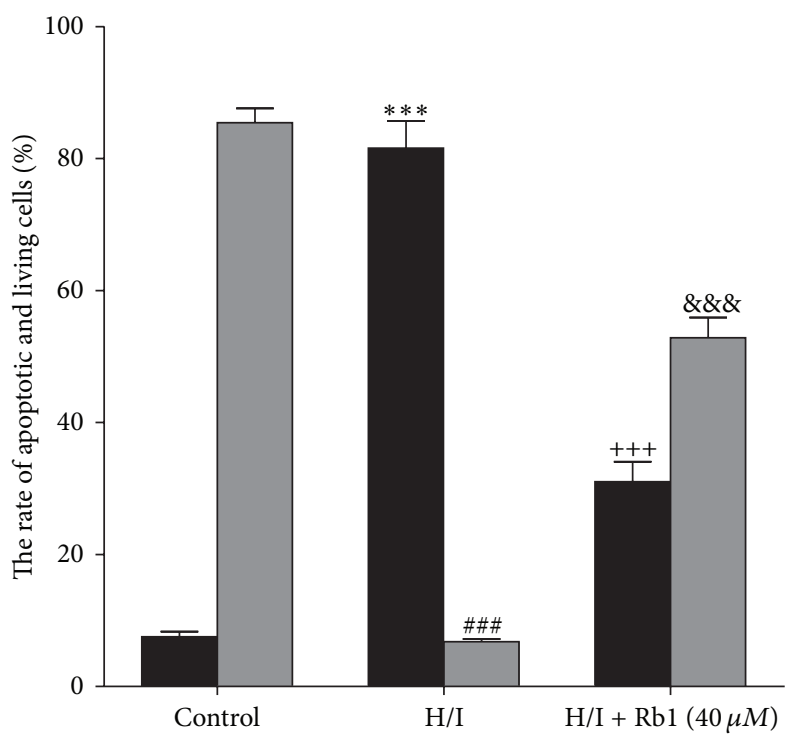

- Apoptotic cells

$\square$ Living cells

(b)

FIGURE 2: Flow cytometry analysis of GS-Rb1 on cell death induced by H/I. (a) NRCMs were cotreated with or without GS-Rb1 (40 $\mu$ M) during H/I for $12 \mathrm{~h}$ and stained with Annexin V-FITC/PI. (b) Quantification of the percent of apoptotic and living cells in each group. Error bars represent mean $\pm \mathrm{SD} .{ }^{* * *} P<0.001$ versus apoptotic cells in control group; ${ }^{\# \# \#} P<0.001$ versus living cells in control group; ${ }^{++} P<0.001$ versus apoptotic cells in H/I group; ${ }^{\& \& \&} P<0.001$ versus living cells in H/I group $(n=3)$. 


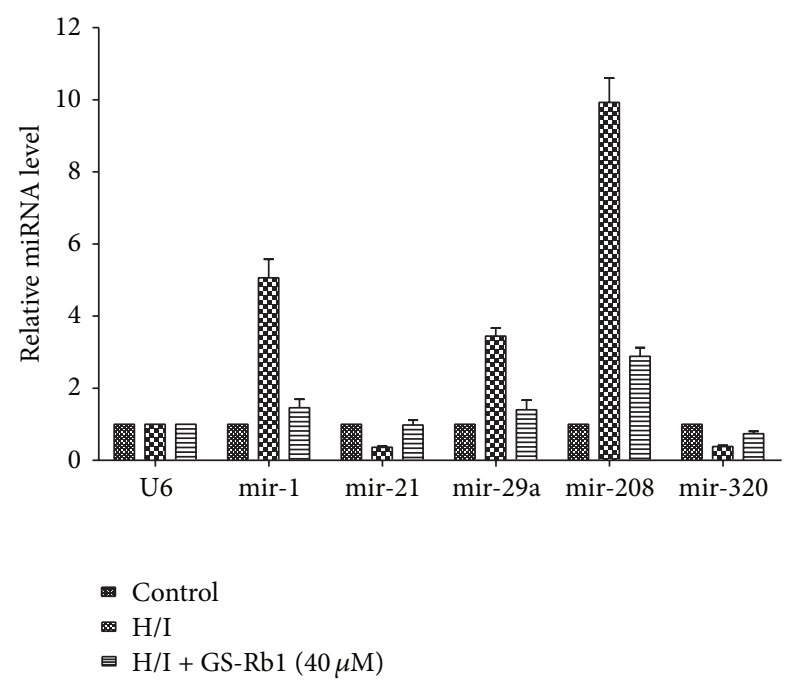

FIGURE 3: Real-time PCR analysis of GS-Rb1 on miRNAs expression change induced by H/I. NRCMs were cotreated with or without GS$\mathrm{Rb} 1(40 \mu \mathrm{M})$ during $\mathrm{H} / \mathrm{I}$ for $12 \mathrm{~h}$. Detection of miRNA expression was performed using poly(A) tailing SYBR Green real-time PCR. The relative expression of miRNA was calculated based on the formula: $2^{-(\Delta \mathrm{CtmiR}-\Delta \mathrm{CtU} 6)}$. Error bars represent mean $\pm \mathrm{SD}$.

hearts subjected to IR injury [28]. Our results demonstrated that GS-Rb1 suppressed the expression of mir-1 and mir-29a in the model group, which might be the microRNA targets of GS-Rb1 to protect cardiomyocytes from $\mathrm{H} / \mathrm{I}$ injuries.

Mir-21 is another cardiac enriched microRNA, which has been proved to be involved in ischemic heart disease, myocardial remodeling, and vascular proliferative diseases [29]. Overexpression of mir-21 plays an important role during cardiomyocytes apoptosis and ischemia/reperfusion- (I/R-) induced heart damages [30, 31]. Mir-320 has the familiar function with mir-21. Mir-320 expression was significantly decreased in the hearts on ischemia/reperfusion in vivo and in vitro. Overexpression of mir-320 enhanced cardiomyocyte apoptosis and increased extent of apoptosis and infarction size in the hearts. Conversely, in vivo treatment with antagomir-320 reduced infarction size and showed cytoprotective [32]. Our results showed that mir-21 and mir-320 significantly decreased in the H/I injured cardiomyocytes and increased by GS-Rb1 treatment following H/I, which suggested that mir-21 and mir-320 might be the potential microRNA targets of GS-Rb1 to protect cardiomyocytes.

Mir-208 is produced exclusively in the heart. A growing number of studies have demonstrated that mir-208 could be selected as a possible biomarker of myocardial injury and myocardial infarction [33,34]. Plasma mir-208 increased significantly after isoproterenol-induced myocardial injury and showed a similar time course to the concentration of $\mathrm{cTnI}$ [35]. Therapeutic inhibition of mir-208a by systemic delivery of antisense oligonucleotide could improve cardiac function and survival during hypertension-induced heart failure [36]. But there was no enough evidence shown that overexpression or knockdown mir-208 was related to the hypoxia/ischemia injuries and cardiomyocytes apoptosis. Our results showed that GS-Rb1 could suppress the expression of mir-208 in model group. The relationship of mir-208 and cardiomyocytes injury or cell apoptosis needs to be further studied.

In conclusion, GS-Rb1 protects hypoxic- and ischemicinduced damaged myocardial cells by regulating expression of miRNAs, which not only is important in uncovering mechanism of GS-Rb1 in hypoxic- and ischemic-induced damage of myocardial cells, but also may provide a new method for treating myocardial diseases in clinical applications.

\section{Conflict of Interests}

The authors declare that they have no conflict of interests.

\section{Authors' Contribution}

$\mathrm{Xu}$ Yan and Jinrong Xue contributed equally to this work.

\section{Acknowledgments}

This work was supported by Grants from the National Natural Science Foundation of China (nos. 81273890 and 81173367) and Postdoctoral Science Foundation of Beijing (2013ZZ-57).

\section{References}

[1] A. Kozomara and S. Griffiths-Jones, "MiRBase: integrating microRNA annotation and deep-sequencing data," Nucleic Acids Research, vol. 39, supplement 1, pp. D152-D157, 2011.

[2] B. K. Dey, K. Pfeifer, and A. Dutta, “The H19 long noncoding RNA gives rise to microRNAs miR-675-3p and miR-675-5p to promote skeletal muscle differentiation and regeneration," Genes \& Development, vol. 28, no. 5, pp. 491-501, 2014.

[3] T. Baars, A. Skyschally, L. Klein-Hitpass et al., "microRNA expression and its potential role in cardioprotection by ischemic postconditioning in pigs," Pflügers Archiv, vol. 466, no. 10, pp. 1953-1961, 2014.

[4] J. Ai, R. Zhang, Y. Li et al., "Circulating microRNA-1 as a potential novel biomarker for acute myocardial infarction," Biochemical and Biophysical Research Communications, vol. 391, no. 1, pp. 73-77, 2010.

[5] G.-K. Wang, J.-Q. Zhu, J.-T. Zhang et al., "Circulating microRNA: a novel potential biomarker for early diagnosis of acute myocardial infarction in humans," European Heart Journal, vol. 31, no. 6, pp. 659-666, 2010.

[6] G. W. Dorn, S. J. Matkovich, W. H. Eschenbacher, and Y. Zhang, "A human 3/ miR-499 mutation alters cardiac mRNA targeting and function," Circulation Research, vol. 110, no. 7, pp. 958-967, 2012.

[7] Z. Liu, J. Chen, W. Huang, Z. Zeng, Y. Yang, and B. Zhu, "Ginsenoside Rb1 protects rat retinal ganglion cells against hypoxia and oxidative stress," Molecular Medicine Reports, vol. 8, no. 5, pp. 1397-1403, 2013.

[8] L. Jia, Y. Zhao, and X.-J. Liang, "Current evaluation of the millennium phytomedicine-ginseng (II): collected chemical entities, modern pharmacology, and clinical applications emanated from traditional chinese medicine," Current Medicinal Chemistry, vol. 16, no. 22, pp. 2924-2942, 2009. 
[9] S.-D. Zheng, H.-J. Wu, and D.-L. Wu, "Roles and mechanisms of ginseng in protecting heart," Chinese Journal of Integrative Medicine, vol. 18, no. 7, pp. 548-555, 2012.

[10] P. Simpson and S. Savion, "Differentiation of rat myocytes in single cell cultures with and without proliferating nonmyocardial cells. Cross-striations, ultrastructure, and chronotropic response to isoproterenol," Circulation Research, vol. 50, no. 1, pp. 101-116, 1982.

[11] S. Tan, F. Zhou, N. Li et al., "Anti-fatigue effect of ginsenoside $\mathrm{Rbl}$ on postoperative fatigue syndrome induced by major small intestinal resection in rat," Biological and Pharmaceutical Bulletin, vol. 36, no. 10, pp. 1634-1639, 2013.

[12] L. Shen, Y. Xiong, D. Q.-H. Wang et al., "Ginsenoside Rb1 reduces fatty liver by activating AMP-activated protein kinase in obese rats," The Journal of Lipid Research, vol. 54, no. 5, pp. 1430-1438, 2013.

[13] N. Lin, D.-L. Cai, D. Jin, Y. Chen, and J.-J. Shi, "Ginseng panaxoside $\mathrm{Rb} 1$ reduces body weight in diet-induced obese mice," Cell Biochemistry and Biophysics, vol. 68, no. 1, pp. 189194, 2014.

[14] T. H. Kang, H. M. Park, Y.-B. Kim et al., "Effects of red ginseng extract on UVB irradiation-induced skin aging in hairless mice," Journal of Ethnopharmacology, vol. 123, no. 3, pp. 446451, 2009.

[15] D. Liu, H. Zhang, W. Gu, Y. Liu, and M. Zhang, "Neuroprotective effects of ginsenoside Rbl on high glucose-induced neurotoxicity in primary cultured rat," PLoS ONE, vol. 8, no. 11, Article ID e79399, 2013.

[16] Z. Liu, J. Chen, W. Huang, Z. Zeng, Y. Yang, and B. Zhu, "Ginsenoside Rb1 protects rat retinal ganglion cells against hypoxia and oxidative stress," Molecular Medicine Reports, vol. 8, no. 5, pp. 1397-1403, 2013.

[17] W. Cheng, D. Wu, Q. Zuo, Z. Wang, and W. Fan, "Ginsenoside $\mathrm{Rbl}$ prevents interleukin-1 beta induced inflammation and apoptosis in human articular chondrocytes," International Orthopaedics, vol. 37, no. 10, pp. 2065-2070, 2013.

[18] J. Wang, L. Qiao, S. Li, and G. Yang, "Protective effect of ginsenoside $\mathrm{Rb} 1$ against lung injury induced by intestinal ischemia-reperfusion in rats," Molecules, vol. 18, no. 1, pp. 12141226, 2013.

[19] J. Wang, L. Qiao, Y. Li, and G. Yang, "Ginsenoside Rb1 attenuates intestinal ischemia-reperfusion-induced liver injury by inhibiting NF- $\kappa \mathrm{B}$ activation," Experimental and Molecular Medicine, vol. 40, no. 6, pp. 686-698, 2008.

[20] Q. Sun, Q.-T. Meng, Y. Jiang, and Z.-Y. Xia, "Ginsenoside Rb1 attenuates intestinal ischemia reperfusion induced renal injury by activating Nrf2/ARE pathway," Molecules, vol. 17, no. 6, pp. 7195-7205, 2012.

[21] Y. Zhang, L. Zhang, W. Chu et al., “Tanshinone IIA inhibits miR-1 expression through p38 MAPK signal pathway in postinfarction rat cardiomyocytes," Cellular Physiology and Biochemistry, vol. 26, no. 6, pp. 991-998, 2010.

[22] L. Zhang, Y. Wu, Y. Li et al., “Tanshinone IIA improves miR-133 expression through MAPK ERK1/2 pathway in hypoxic cardiac myocytes," Cellular Physiology and Biochemistry, vol. 30, no. 4, pp. 843-852, 2012.

[23] Y. Zhao, J. F. Ransom, A. Li et al., "Dysregulation of cardiogenesis, cardiac conduction, and cell cycle in mice lacking miRNA1-2," Cell, vol. 129, no. 2, pp. 303-317, 2007.

[24] Z.-X. Shan, Q.-X. Lin, C.-Y. Deng et al., "MiR-1/miR-206 regulate Hsp60 expression contributing to glucose-mediated apoptosis in cardiomyocytes," FEBS Letters, vol. 584, no. 16, pp. 3592-3600, 2010.

[25] B. He, J. Xiao, A.-J. Ren et al., "Role of miR-1 and miR-133a in myocardial ischemic postconditioning," Journal of Biomedical Science, vol. 18, no. 1, article 22, 2011.

[26] Z. Pan, X. Sun, J. Ren et al., "miR-1 exacerbates cardiac ischemiareperfusion injury in mouse models," PLoS ONE, vol. 7, no. 11, Article ID e50515, 2012.

[27] S.-Y. Park, J. H. Lee, M. Ha, J.-W. Nam, and V. N. Kim, "miR29 miRNAs activate p53 by targeting p $85 \alpha$ and CDC42," Nature Structural \& Molecular Biology, vol. 16, no. 1, pp. 23-29, 2009.

[28] Y. Ye, Z. Hu, Y. Lin, C. Zhang, and J. R. Perez-Polo, "Downregulation of microRNA-29 by antisense inhibitors and a PPAR$\gamma$ agonist protects against myocardial ischaemia-reperfusion injury," Cardiovascular Research, vol. 87, no. 3, pp. 535-544, 2010.

[29] Y. Cheng and C. Zhang, "MicroRNA-21 in cardiovascular disease," Journal of Cardiovascular Translational Research, vol. 3, no. 3, pp. 251-255, 2010.

[30] C. Yin, X. Wang, and R. C. Kukreja, "Endogenous microRNAs induced by heat-shock reduce myocardial infarction following ischemia-reperfusion in mice," FEBS Letters, vol. 582, no. 30, pp. 4137-4142, 2008.

[31] C. Wei, L. Li, I. K. Kim, P. Sun, and S. Gupta, "NF- $\kappa$ B mediated miR-21 regulation in cardiomyocytes apoptosis under oxidative stress," Free Radical Research, vol. 48, no. 3, pp. 282-291, 2014.

[32] X.-P. Ren, J. Wu, X. Wang et al., "MicroRNA-320 is involved in the regulation of cardiac ischemia/reperfusion injury by targeting heat-shock protein 20," Circulation, vol. 119, no. 17, pp. 2357-2366, 2009.

[33] E. Boštjančič, N. Zidar, D. Štajer, and D. Glavač, "MicroRNAs miR-1, miR-133a, miR-133b and miR-208 are dysregulated in human myocardial infarction," Cardiology, vol. 115, no. 3, pp. 163-169, 2010.

[34] Y. Cheng, X. Wang, J. Yang et al., "A translational study of urine miRNAs in acute myocardial infarction," Journal of Molecular and Cellular Cardiology, vol. 53, no. 5, pp. 668-676, 2012.

[35] X. Ji, R. Takahashi, Y. Hiura, G. Hirokawa, Y. Fukushima, and N. Iwai, "Plasma miR-208 as a biomarker of myocardial injury," Clinical Chemistry, vol. 55, no. 11, pp. 1944-1949, 2009.

[36] Y. Cheng, X. Wang, J. Yang et al., "A translational study of urine miRNAs in acute myocardial infarction," Journal of Molecular and Cellular Cardiology, vol. 53, no. 5, pp. 668-676, 2012. 


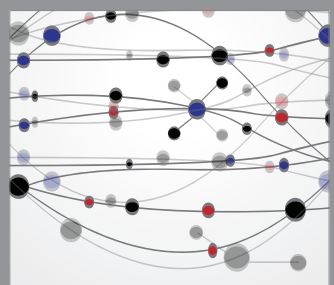

The Scientific World Journal
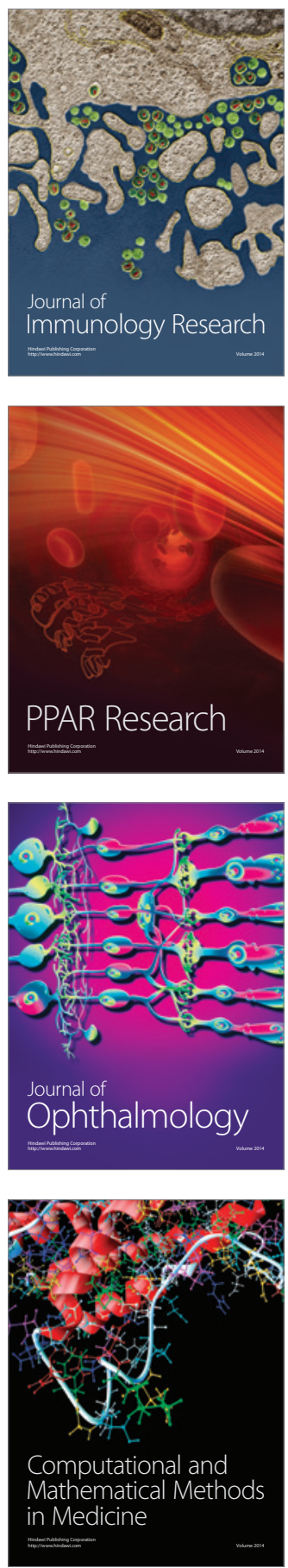

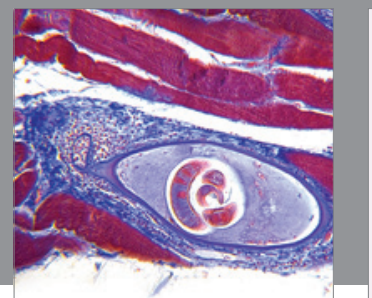

Gastroenterology

Research and Practice
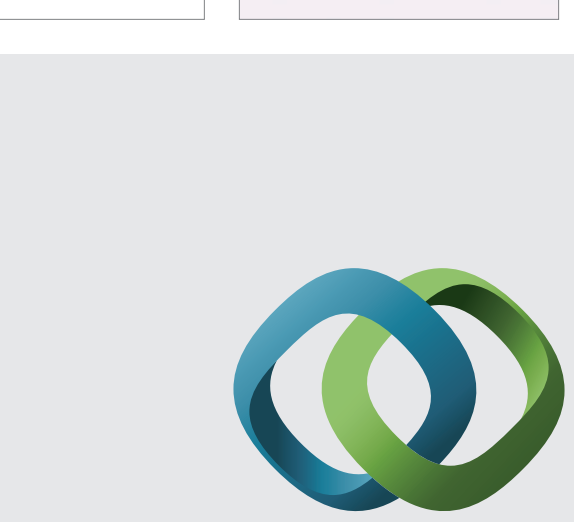

\section{Hindawi}

Submit your manuscripts at

http://www.hindawi.com
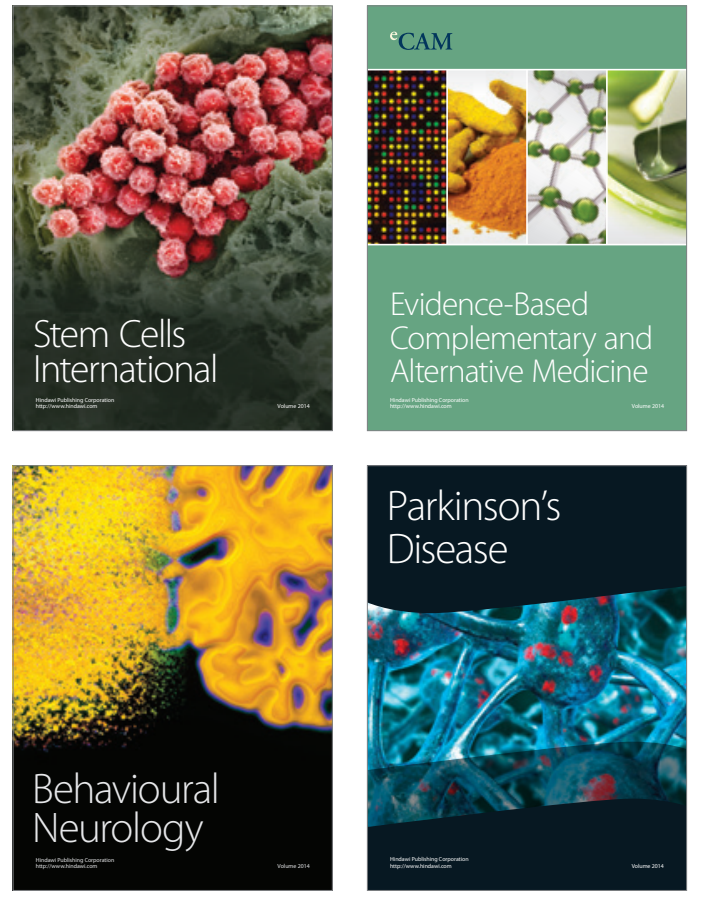
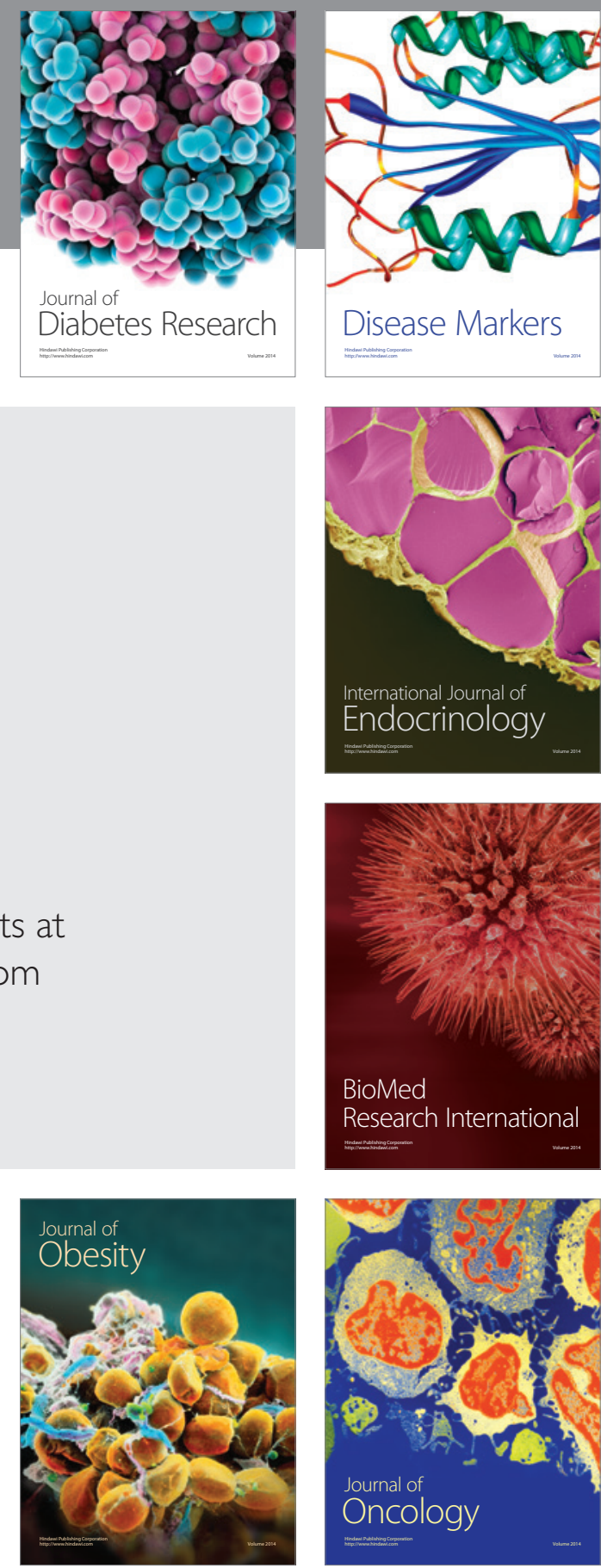

Disease Markers
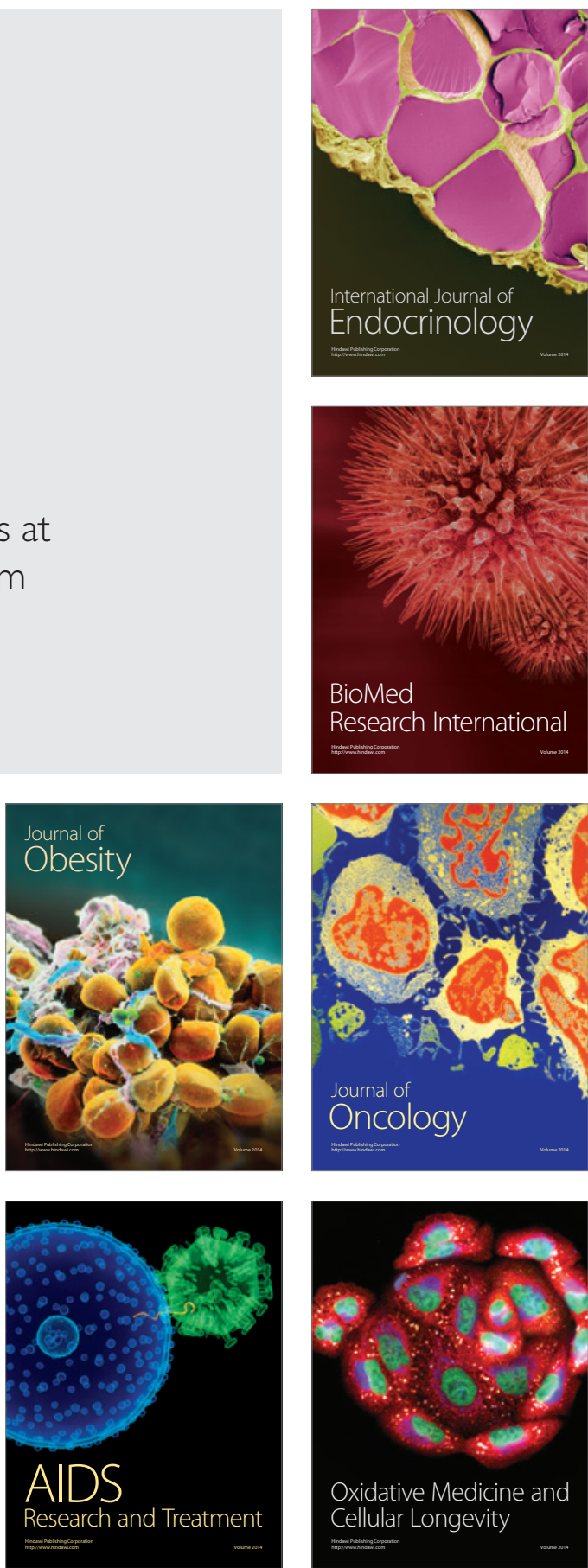